\title{
Nutrition of women with hair loss problem during the period of menopause
}

\author{
Zuzanna Sabina Goluch-Koniuszy \\ Department of Human Nutrition Physiology, West Pomeranian University of Technology in Szczecin, Poland
}

\begin{abstract}
During the period of menopause as an effect of changes in hormone status, one of the most common ailments for women is hair loss. Taking into consideration fact that the ingredients of diet contained in various groups of consumed food products are both precursors in steroid hormones synthesis as well as have direct impact on structure, growth and keeping hair in skin integument, this is the reason why nourishing support for women during this period of life as well as during the hair loss therapy is reasonable.

Standard value proteins containing Sulphur amino-acids: cysteine and methionine as precursor to keratin hair protein synthesis are basic element of diet conditioning of hair building. Irreplaceable having impact on keeping hair in skin integument is exogenous L-lysine, mainly present in the inner part of hair root is responsible for hair shape and volume. Fats present in the diet take part in steroid hormones synthesis (from cholesterol) thus have influence on keeping hair in skin integument. Women diet should contain products rich in complex carbohydrates, with low glycemic index and load containing fiber regulating carbohydrate-lipid metabolism of the body. Vitamins also have impact on the state of hair: $\mathrm{C}$ vitamin, group $\mathrm{B}$ and $\mathrm{A}$ vitamins. Minerals which influence hair growth are: $\mathrm{Zn}, \mathrm{Fe}, \mathrm{Cu}, \mathrm{Se}, \mathrm{Si}, \mathrm{Mg}$ and $\mathrm{Ca}$. It is worthwhile to pay closer attention to diet in women who besides hormone changes and undertaken pharmacotherapy are additionally exposed to chronic stress and improperly conducted cosmetic's and hairdresser's treatments.
\end{abstract}

Key words: nourishing support, hair loss, menopause.

\section{Introduction}

Shining and healthy hair is the attribute of healthy people looking after themselves and taking good care of their body, but for women they are also a decoration which gives them sense of wellbeing. However, weak, falling out hair might extremely lower the comfort of women's life, lead to lowering of self-esteem and stress which is bound to this may even intensify the problem.

In physiological conditions on head there is $80 \%$ of hair in the course of growing (anagen), 1\% in the period of involution of hair follicle (catagen) and the rest of percentage is hair in the rest period (telogen). Depending on various factors, the number and density of women hair might change not only during illness but also in physiological conditions, among others in the period of menopause [1]. Daily loss of hair should not exceed 70-100 hair, but it becomes a problem when the loss is higher than 100 daily during the period longer than few weeks.

Physiologically, during the period of pre menopause in women density of estrogen in blood lowers with natural rise of androgen concentration, which might be the cause of androgenic hair loss. Hair loss in women usually appears as a spread thinning of hair mainly in cen- tral and forehead part, and sometimes also in parietal and occipital part [2]. During the occurrence before last menstruation, menstruation cycles start to be irregular and extended. Characteristic for this time is change in sex hormone concentration in different ways in various women. Some skin and hair diseases during the premenopause period might be the effect of illness tied to excess production of androgens by adrenal gland or ovaries and/or tissue. Women with higher concentration of these hormones, developed by endo- or exogenous factors lose hair [3]. Dihydtrotestosterone (DHT) originating from testosterone with the participation of $5 \alpha$-reductase present in hair follicles is a hormone which in the strongest way influences hair follicles. It is stronger by 2.5 times than testosterone. DHT hormone weakens hair follicles and leads to hair falling out.

The problem of hair loss in women during the period of menopause needs precise diagnosing and next undertaking a proper cure [2]. Almost $20-60 \%$ of women before reaching the age of 60 suffer from this [4]. However taking into consideration fact that main cause of hair loss by women during this period of life are changes in hormone metabolism of the body that is why it is reasonable to nutritiously support women. Ingredients of diet contained in various groups of groceries are both 
precursors in steroid hormone synthesis as well as have direct impact on build, growing and keeping hair in skin integument.

\section{Essential nutrients}

\section{Proteins}

Standard value proteins containing Sulphur aminoacids: cysteine and methionine as precursor to keratin hair protein synthesis are basic element of diet conditioning of hair building. Protein or protein calorific malnutrition causes disturbed hair synthesis (hair fragility and brittleness), their weakness (they are in the form of lanugo) and hair loss [5]. Hair follicles can be atrophic. Keratin, the main ingredient of hair shaft and hair shield, is produced in cells called keratinocytes in basic and horny layer of epidermis. Whereas spirally twisted keratin fibers, present in the inside of hair shaft (cortex of hair shaft), are responsible for hair elasticity. Keratin found in the hair shield gives them continuity and shine, so with its lack hair show tendency to divide into two parts [6].

Cysteine, as keratin ingredient occurs in hair in the highest amount (10-17\%) and is one of the endogenous amino acids which synthesis is addicted to methionine presence. The rate of growth, hair diameter and protein synthesis depend on cysteine. Active form of $B_{6}$ vitamin (pyridoxal phosphate) increases L-cysteine incorporation to keratin [7]. Also essential amino acid having impact on keeping hair in skin integument is exogenous L-lysine, mainly present in the inner part of hair root is responsible for hair shape and volume. With insufficient supply of lysine in food, hair becomes brittle, thin and limp. This amino acid has significant impact on zinc and iron absorption [8].

Proteins should make $10-15 \%$ of energy value of the (WED) diet in the amount of ( $0.9 \mathrm{~g} / \mathrm{kg}$ of body mass per day), and it is recommended they should be consumed mainly in I and II breakfast, dinner and in smaller portions as afternoon snack and supper [9]. Good sources of above mentioned amino acids in women's diets should be cottage cheese, yoghurt, fish, meat (veal, beef), poultry (turkey, chicken), legume seeds (soya, lentils, beans, peas, broad beans), seeds (pumpkin and sunflower seeds, sesame), nuts (pistachio, peanuts), grain products (buckwheat, barley groats, hulled barley, brown rice, rye whole-meal bread and graham bread). Additional source of these amino acids are eggs, it is recommended to consume 2-3 eggs per week.

\section{Fots}

Fats present in the diet take part in steroid hormones synthesis (from cholesterol) thus have influence on keeping hair in skin integument. Saturated fatty ac- ids also contained in the diet, might increase this state by increasing sebum secretion. "Protective coat" for skin integument and its products in the form of lipid complexes are ceramides, sterols and phosphor lipids and also free fatty acids. Deficiency of these compounds in women's body causes decrease in hair hydration, even to their loss as a result of improper state of the hair bulbs. Low consumption of linoleic and linolenic acids and long-chain polyunsaturated fatty acids, which are important ingredients of horny layer of epidermis, causes to lose hair. Fatty acids from the omega-3 polyunsaturated fatty acids (EPA and DHA) family are found mainly in fish, flax seeds, walnuts, wheat sprouts. Omega- 6 polyunsaturated fatty acids, present in plant oils, are also necessary for keeping proper build of hair, however their excess in the diet may lead to immunological balance disorders of the body and higher risk of inflammation states, which could cause hair asthenia and their loss [10]. Fats should constitute 25-35\% WED and their source should be fish, poultry, eggs, olive oil and rapeseed oil $[9,11]$.

\section{Carbohydrates}

Carbohydrates also influence the state of hair. It has been shown that consumption of highly processed foods, rich in simple sugars is one of the indirect factors causing excess hair loss. Diet rich in simple sugars stimulates sebum secretion by sebaceous glands. In physiological conditions sebum has a beneficial impact on hair, however if the secretion is too high it becomes food for microorganisms found on skin, which cause decomposition of triacylglycerols contained in them. It leads to release of fatty acids irritating and causing development of inflammation state [12]. Produced this way excess amount of fatty acids causes secondary increase of sebum secretion that leads to keratinization disorders.

What is more, diet rich in simple sugars implies improper glycaemia and insulinemia which directly contributes to increase in of synthesis of ovarian androgens and indirectly through suppression of SHBG synthesis in liver and type 1 protein bonding insulin-like growth factor (IGF-1). In hair follicle insulin has direct impact on hair growth and increase of DHT concentration which leads to miniaturization [13]. Insulin also has impact on circulatory disturbances in blood vessels nourishing the scalp leading to local hypoxia which contributes to hair loss [14]. This is the reason why women diet should contain products rich in complex carbohydrates, with low glycemic index and load [15] containing fiber regulating carbohydrate-lipid metabolism of the body [16]. Carbohydrates should form 50-70\% WED, including no more than $10 \%$ WED originating from sucrose $[9,17]$. The source of carbohydrates in diet should be full grain breads, grits, rice, whole meal pasta, vegetables and fruit with low glycemic load. 


\section{Vitamins}

Vitamins also have impact on the state of hair: $C$ vitamin, group $B$ and $A$ vitamins. Insufficient supply of $C$ vitamin in diet has influence on creating the hair shaft. Increased activity of hair follicle channel cells can be observed and the excess production of keratin starts blocking its orifice which results in characteristic very curly hair [18]. Avitaminosis of ascorbic acid might be indirect cause of telogenic baldness. Vitamin C increases absorption of non-heme iron, originating from plant products. Products containing vitamin C are: vegetables (green parsley leaves, kale, horseradish, peppers, Brussels sprouts, broccoli, cauliflower, spinach and savoy) and fruit (black currants, strawberries, wild strawberries, kiwi, red currants and citrus fruit) [11].

Rasheed et al. [19] pointed out at low concentration of $D_{2}$ vitamin in serum of women aged 18-45 with prolonged or telegenic hair loss. Vitamin D promotes hair follicle differentiation, without affecting proliferation.

Basic source (endogenous) of D vitamin in the body is synthesis of its precursor on skin (mainly in the prickle layer of epidermis) with 7-dehydrocholesterol under the influence of UVB (290-315 nm). Next from this compound, as the result of isomerization, emerges stable cholecalciferol ( $D_{3}$ vitamin). However in the situation when period of time in the open air and contact with UV rays is limited, the diversified diet should compensate the lowered synthesis of 7-dehydrocholesterol in skin and lower hydroxylation of D vitamin in kidneys $[20,21]$.

The best source of $D_{3}$ vitamin in women's diet should be fat fish (marcel, salmon, sardines), whale or tuna liver oil, and also products containing lower amounts such as meat, poultry, eggs and full fat diary. Consumption of mushrooms and yeast will additionally supply body in $\mathrm{D}_{2}$ (ergocalciferol).

Vitamin $D_{3}$ from skin synthesis, as well as the one from the consumed food, together with the transporting protein goes by blood vessels to liver, kidneys and other organs, where it is hydroxylated. The effect of this process is active metabolite $1.25(\mathrm{OH}) 2 \mathrm{D}_{3}$ [22]. Supplying vitamins from both sources has special meaning for women with excess body mass because leptin produced by fat tissue leads to lowering vitamin $D$ conversion to $25(\mathrm{OH}) \mathrm{D}$ and in consequence $1.25(\mathrm{OH})_{2} \mathrm{D}_{3}$. Overweight women are susceptible to lowered bioavailability $1.25(\mathrm{OH})_{2} \mathrm{D}_{3}$ that is the reason why its lowered concentration in blood serum is being observed $[23,24]$.

The studies on vitamin $D_{3}$ metabolism led to identifying in intestinal extracts specific receptor VDR (vitamin $D$ receptor), being the part of family of steroid receptors. Complex 1.25(OH) ${ }_{2} \mathrm{D}_{3} / \mathrm{VDR}$ works as transcription factor and is necessary for keeping hair in proper condition. In the studies on model animals it was shown that activation of VDR receptor plays significant role in the cycle of hair follicle, especially in anagen initiation and is independent of calcium and phosphorus content in diet [25].

Folates take part in producing red blood cells and hemoglobin, which role is to transport oxygen, among others, to tissues building hair. What is more folate are responsible for stimulating of rebuilding of hair follicle cells, prevent hair graying and falling out and regulate sebum glands functioning [26]. The source of folate in women's diet should be: kale, brussels sprouts, green peas, dry peas, white beans, asparagus, beets, kohlrabi halibut, cod but also in small amounts consumed eggs and poultry liver [11].

Pantothenic acid ( $B_{5}$ vitamin) prevents too early hair graying and can restore their natural color. It is also responsible for proper hair growth due to its part in cell division in hair follicle and gives hair proper moisture, has anti-inflammatory properties, protects, has moisturizing abilities, regulates functioning of sebum glands and accelerates melanin creating [26]. Products rich in $B_{5}$ vitamin that is mixture of pantothenic acid, pantein, pantenol and coenzyme $A$ are: mushrooms, cauliflower, liver, soya, hen eggs, and baking yeast, whole grains, beans, milk and green leafy vegetables [11].

Biotin ( $B_{7}, H$ vitamin) is a vitamin taking part in fat and protein metabolism and its deficiency might lead to hair loss. Thanks to the presence of Sulphur it influences the state of skin integument lowering sebum secretion and activates hair growth. Long term nourishment biotin deficiency leads to fatty acids metabolism (increase of palmitic acid concentration in liver and cholesterol concentration in blood), which symptoms are erythematous and seborrheic skin inflammation (conjunctivitis, greasy hair, hair loss and nails brittleness) [27]. Biotin can be found in groceries of plant origin (mainly in cereal germs) and animal origin. In the free form the biggest amount_can be found in milk and in vegetables, and in the bound form in meat, liver, egg yolk, yeast and some nuts [11].

Niacin (PP vitamin) influences water transformation of skin, causes angiectasia, detoxifies skin and is necessary for keeping hair in proper state [28]. Main source of niacin in diet should be: meat, whole wheat grains, legume vegetables, seeds, milk, green leafy vegetables, fish, peanuts, shellfish and yeast [11].

Cobalamin ( $B_{12}$ vitamin) may have connection to excess hair loss in woman with anemia depression [29]. The sources of $B_{12}$ are animal products: meat and processed meats, fish, eggs, milk and dairy products and seafood.

Deficiency of A vitamin in diet may cause decrease in cycle speed of cell regeneration and synthesis. Because of its solubility in fats, A vitamin is responsible for moisturizing and protects hair giving them resistance from being fragile. However its excess, coming from animal products cumulates in liver it can also be the cause of hair fall (which may result from dysregulated immune function) [30], that is the reason why a good 
source is its form coming from carotenoids present in vegetables and fruit.

\section{Minerals}

Minerals which influence hair growth are: $\mathrm{Zn}, \mathrm{Fe}, \mathrm{Cu}$, Se, Si, Mg and Ca.

Zinc takes part in carbohydrates, proteins and fats metabolism and at the same time influences hair follicles and hair growth. It is enzyme activator stimulating protein transformation, which have sulfide bonds, necessary for hair building. Its proper concentration in blood should be $70-250 \mu \mathrm{g} / \mathrm{dl}$, so we do not observe its clinical deficiencies. Research shows its influence on hair cycle [31]. Besides this, zinc influences A vitamin keratinizing hair. Its deficiency in diet suppresses hair growth, can lead to telogen effluvium, thin white and brittle hair and cause hair fall [32], especially in women using diuretic drugs.

Iron deficiency has been associated with hair loss as in alopecia areata, androgenetic alopecia, and telogen effluvium [33]. Iron as hemoglobin component supplies energy to matrix cells being subject to division and differentiation which results in hair production. Influences enzymes work responsible for hair build and growing rate. Iron is a cofactor for ribonucleotide reductase of enzyme limiting DNA synthesis speed that is why its deficiency contributes to inhibition proliferation. Its capturing and use is influenced by presence of L-lysine in diet. Especially in women with recognized anemia and ferritin concentration in blood hair is thin, brittle and falls out [34]. Ferritin levels less than $40 \mathrm{ng} / \mathrm{ml}$ are associated with an increase of telogen phase. When the levels are between $40 \mathrm{ng} / \mathrm{ml}$ and $70 \mathrm{ng} / \mathrm{ml}$, an excess of telogen hair loss is also observed [35].

The best sources of this element are animal products containing better assimilated heme iron (beef and pork, poultry, pork and lamb liver, fish) but valuable diet variety are plant products such as: soya, white beans, pasticcio nuts, green parsley leaves, dried apricots and figs [19, 33].

Copper is crucial for aminoxydases required for oxidation of thiol groups to dithio- crosslinks, are essential for keratin fiber strength. Copper has a stimulating effect on the proliferation of keratinocytes and fibroblasts in monolayers, has a vital role in the activation of key enzyme systems specific to tissue formation and repair, and participates in the cross-linking and maturation of collagen in healing wounds [36].

Copper, present in the same products as iron contributes to hair strengthening by influencing growth of proper horn like hair structure. Its deficiency in diet might be the cause of lowered number of sulfur bridges responsible for strength and elasticity of hair resulting in brittle, weak, curly hair with tendency to early graying [6].

Selenium is an essential component of Selenium proteins playing an important role in many biological functions, such as antioxidant defense, formation of thyroid hormones, DNA synthesis, fertility and reproduction [37]. Hair receives trace elements mostly from the blood and is able to uptake selenium in its matrix during keratinization. Selenium is a component of at least 35 proteins many of which are enzymes and with its deficiency in the body hair loss with pseudo albinism occurs [38]. Although much less common than selenium deficiency, selenium toxicity can affect individuals as a result of over supplementation, which results in hair loss. The main food groups providing selenium in the diet are bread and cereals, meat, liver, fish (cod, canned tuna), eggs, and milk/dairy products. Fruit and vegetables typically contain relatively small amounts of selenium [39].

Silicon is responsible for growth and shine of hair [40]. It can be found in food in the form of silicon acid in grains and their whole meal products (grits, rice, oatmeal and bran, whole grain breads) in garlic, chives and seafood.

Magnesium, taking part in protein transformation, is responsible for division, growth and ripening processes of cells, taking into consideration its part in immunological reactions, protecting and alleviating inflammation states which causes its deficiency have direct or indirect contribution to hair fall. The source of magnesium in women's diet should be cacao, grits, whole grain breads, nuts and legumes [11, 41].

Calcium is also an element playing significant role in keeping hair in proper state and women in particular in the perimenopause period are exposed to its deficiency [42]. Concentration of Calcium in hair exceeds by 200 times concentration of Calcium in blood serum and erythrocytes. As studies of Wlaźlak et al. [42] show significant decrease of this element concentration in hair starts in women at the age of 49. According to WHO expert panel has concluded that this value is $520 \mathrm{mg}$ per day for adults living in a western environment and consuming a western diet [43]. The extra urinary calcium loss during the postmenopausal period is $30 \mathrm{mg}$ per day. As calcium absorption in this age group is lower than young adulthood, the amount of extra dietary intake needed to offset this loss is as high as $260 \mathrm{mg}$. This would increase the recommended intake from $1000 \mathrm{mg}$ for younger adults to $1300 \mathrm{mg}$ per day. Because interspersed sources of this element in diet should be first of all dairy products containing lactose facilitating its absorption and also soya, parsley green leaves, hazelnuts, white beans, kale, walnuts, fish and cabbages [11].

\section{Other compounds}

\section{Polyphenols}

Polyphenols are the most abundant antioxidants in human diets and the largest and best studied class of 
polyphenols is flavonoids. It a group which diversified structural construction of relations contains: flavanones, flavanoles, flavones and isoflavones, flavonoles and anthocyanins. These relations among others play the role of plant hormones, growth regulators and inhibitors of many enzymatic reactions [44]. Flavonides directly in the stratum corneum of skin act anti radically and antioxidant. In deeper parts of skin they modify enzyme activity and protect from harmful properties of UV radiation. In the corium they influence the state of blood vessels and stimulate skin micro circulation which nourishes hair. Indirectly flavonoids increase absorption from the alimentary tract of vitamin $C$ and protect functions conducted by it. It was presented that flavonoids contained in green tea stimulate growth of hair follicles by lengthening their anagen phase [45]. However in the research conducted on model animals was shown that flavonoids contained in herbs inhibiting $5 \alpha$-reductase contributed to faster hair regrowth [46].

Despite the fact that the highest number of flavonoid compounds can be found in bitter chocolate ( $>70 \%$ of cocoa) the main source of flavonoids in women diet should be vegetables (onions, tomatoes, peppers, broccoli) and fruit (apples, berries, black currant, citrus fruits and grapes). Flavonoids also can be found in some grains, seeds of lentils, spices and red wine, green tea, coffee, tea and cocoa [47].

The main classes phytoestrogens (structurally and functionally similar to mammalian estrogens) found in the human diet are isoflavones (daidzein, genistein, and glycitein), lignans (enterodiol and enterolactone). The primary food sources of isoflavones are soy and soy products, and sources for lignans are cereals, flaxseed, and berries. These compounds delay the menopause in women and relieve its consequences [48].

\section{Liquids}

Water influences the hydration degree and state of hydro lipid layer on its surface. Physiologically, the amount of water contained in the body which is bounded in skin amounts to $20 \%$ [49]. That is why mineral and spring waters containing minerals are a very good source of water and minerals. Liquids should be consumed in the amount $30-35 \mathrm{ml} / \mathrm{kg}$ of body mass/day including $1.5 \mathrm{I}$ in the form of water (boiled, mineral) best between meals. Other sources of liquids during the day could be also compotes, unsweetened juices, tea, Inka coffee, coffee made from wheat, natural coffee and soups.

\section{Supplementation}

The use of nutritional supplements to correct nutritional deficiencies can undisputedly help many patients and improve quality of life. Dermatologists in skin build and function disorders and in case of hair loss most often recommend diet supplementation with preparation containing $\mathrm{Zn}, \mathrm{Cu}$, Fe (especially for vegetarian people), Vitamins A, D, E, niacin, $B_{12}$ and biotin. Typically, they are based on the B-complex group, including pantothenic acid and p-aminobenzoic acid. For example positive influence of six months supplementation with L-lysine (1.5 g/day) and iron (72 mg/day) on increase of ferritin watching in blood serum of women with chronic telogen effluvium and $36 \%$ lower hair fall, which sooner reacted to supplementation with only iron [26], was observed.

Orthosilicic acid increased hair tensile strength and thickness in a controlled study after 6 months and decreased hair brittleness in a study after 20 weeks [40]. In a randomized, placebo-controlled study in 30 women, a supplement of L-cysteine in combination with medicinal yeast and pantothenic acid led to a normalization of the rate of anagen after 6 months [50].

However, supplementation of diet should take place only for medical reasons, under control and till the achieving the improvement of the state of body saturation with vitamins or mineral content. Significant amount contained in supplements (in amounts above the recommended dietary allowance RDA) the content of vitamins and mineral ingredients may cause in women who take them unnecessary phenomenon of hyperalimentation, exceeding safe dosage and cause unfavorable symptoms of their excess amount. During treatment, zinc levels should be monitored because overdose can lead to Copper or Calcium deficiency, drowsiness, and headache. In case of using multiple ingredients supplements often content of Zinc to Iron are equalized (recommended Fe : $\mathrm{Zn}=3: 1$ ) which causes decrease in Iron uptake.

The most likely mechanism for Zinc-induced Copper deficiency can be explained by the competitive absorption between Zinc and Copper in enterocytes of the small intestine. Excess Zinc intake stimulates the production of metallothionein. Copper, however, has a higher affinity for metallothionein, so it displaces Zinc and is subsequently excreted, leading to hypocupremia [51].

Summing up it is the variety of women's diet in the period of menopause in above described groceries because only a balanced diet that can prevent deficiency of ingredients taking part in hair building process and keeping it in skin integument. It is worthwhile to pay closer attention to diet of women who besides hormone changes and undertaken pharmacotherapy are additionally exposed to chronic stress and improperly conducted cosmetic's and hairdresser's treatments.

\section{Disclosure}

Authors report no conflict of interest. 


\section{References}

1. Miziołek B, Brzezińska-Wcisło L, Wcisło-Dziadecka D, et al. Trichological problems related to menopause. Post Nauk Med 2015; 28: 211-216.

2. Herskovitz I, Tosti A. Female pattern hair loss. Int J Endocrinol Metab 2013; 11: e9860.

3. Imko-Walczuk B, Cegielska A, Głombiowska M. Changes in hair distribution in postmenopausal women. Przegl Derm 2012; 99: 62-67.

4. Shapiro J. Clinical practice: Hair loss in women. N Engl J Med 2007; 357: 1620-1630.

5. Jen $M$, Yan AC. Syndromes associated with nutritional deficiency and excess. Clin Dermatol 2010; 28: 669-685.

6. Markiewicz-Żukowska R. Supplements for hair health. Med Estet Anti Aging 2010; 2: 31-35.

7. D’Agostini F, Fiallo P, Pennisi TM, De Flora S. Chemoprevention of smokeinduced alopecia in mice by oral administration of L-cystine and vitamin B6. J Dermatol Sci 2007; 46: 189-198.

8. Rushton DH, Norris MJ, Dover R, Busuttil N. Causes of hair loss and the developments in hair rejuvenation. Int J Cosmet Sci 2002; 24: 17-23.

9. Jarosz M. Nutrition Standards for the Polish population - amendment. Instytut Żywności i Żywienia, Warszawa 2012.

10. Pappas A. Epidermal surface lipids. Dermatoendocrinol 2009; 1: 72-76.

11. Kunachowicz H, Nadolna I, Przygoda B, Iwanow K. Food composition tables. PZWL, Warszawa, 2005.

12. James MJ, Gibson RA, Cleland LG. Dietary polyunsaturated fatty ac ids and inflammatory mediator production. Am J Clin Nutr 2000; 71 (1 suppl): 343S-348S.

13. Nabaie L, Kavand S, Robati N, et al. Androgenic alopecia and insulin resistance: are they realy related? Clin Exp Dermatol 2009; 34: 694-697.

14. Matilainen V, Laakso M, Hirsso P, et al. Hair loss, insulin resistance, and heredity in middle-aged women. A population-based study. J Cardiovasc Risk 2003; 10: 227-231.

15. Monro JA, Shaw M. Glycemic impact, glycemic glucose equivalents, glycemic index and glycemic load: definitions, distinctions, and implications. Am J Clin Nutr 2008; 87: 237-243.

16. Weickert MO, Pfeiffer AFH. Metabolic effects of dietary fiber consumptional and prevention of diabetes. J Nutr 2008; 138: 439-442.

17. WHO. Sugars intake for adult and children Guideline. WHO, Geneva 2015.

18. Ragunatha S, Inamadar AC, Palit A, et al. Diffuse nonscarring alopecia of scalp: An indicator of early infantile scurvy? Ped Derm 2008; 25: 644 646.

19. Rasheed H, Mahgoub D, Hegazy R, et al. Serum ferritin and vitamin d in female hair loss: do they play a role? Skin Pharmacol Physiol 2013; 26: 101-107.

20. Amor KT, Rashid RM, Mirmirani P. Does D matter? The role of vitamin D in hair disorders and hair follicle cycling. Dermatol Online J 2010; 16: 3.

21. Lehmann B. Role of vitamin D3 pathway in healthy and diseased skinfacts, contradictions, and hypotheses. Exp Dermatol 2009; 18: 97-108.

22. Bouvard B, Annweiler C, Salle A, et al. Extraskeletal effects of vitamin D: facts, uncertainties and controversies. Joint Bone Spine 2011; 78 : 10-16.

23. Florez $\mathrm{H}$, Martinez R, Chacra W, et al. Outdoor exercise reduces the risk of hypovitaminosis D in the obese. J Steroid Biochem Mol Biol 2007; 103: 679-681.

24. Maetani M, Maskarinec G, Franke AA, Cooney RV. Association of leptin, 25-hydroxyvitamin D, and parathyroid hormone in women. Nutr Cancer 2009; 61: 225-231.

25. Bolland MJ, Ames RW, Grey AB, et al. Does degree of baldness influence vitamin D status? Med J Aust 2008; 189: 674-675.

26. Rushton DH, Norris MJ, Dover R, Busuttil N. Causes of hair loss and the developments in hair rejuvenation. Int J Cosmet Sci 2002; 24: 17-23.

27. Said HM. Cell and molecular aspects of human intestinal biotin absorption. J Nutr 2009; 139: 158-162.

28. Draelos ZD, Jacobson EL, Kim H, et al. A pilot study evaluating the efficacy of topically applied niacin derivatives for treatment of female pattern alopecia. J Cosmet Dermatol 2005; 4: 258-261

29. Schmitt JV1, Ribeiro CF, Souza FH, et al. Hair loss perception and symptoms of depression in female outpatients attending a general dermatology clinic. An Bras Dermatol 2012; 87: 412-417.
30. Shih MY, Kane MA, Zhou P, et al. Retinol Esterification by DGAT1 Is Essential for Retinoid Homeostasis in Murine Skin. J Biol Chem 2009; 284: 4292-4299.

31. Kil MS, Kim CW, Kim SS. Analysis of serum zinc and copper concentrations in hair loss. Ann Dermatol 2013; 25: 405-409

32. Yanagisawa $\mathrm{H}$. Zinc deficiency and clinical practice - validity of zinc preparations. Pharm Soc Jpn 2008; 128: 333-339.

33. Trost LB, Bergfeld WF, Calogeras et al. The diagnosis and treatment of iron deficiency and its potential relationship to hair loss. J Am Acad Dermatol 2006; 54: 824-844.

34. Park SY, Na SY, Kim JH, et al. Iron plays a certain role in patterned hair loss. J Korean Med Sci 2013; 28: 934-938.

35. Olsen EA. Female pattern hair loss. J Am Acad Dermatol 2001; 45: S70$\mathrm{S} 80$.

36. Driscoll MS, Kwon EK, Skupsky H, et al. Nutrition and the deleterious side effects of nutritional supplements. Clin Dermatol 2010; 28: 371-379.

37. Mehdi Y, Hornick J-L, Istasse L, Dufrasne I. Selenium in the environment, metabolism and involvement in body functions. Molecules 2013; 18: 3292-3311.

38. Masumoto K, Nagata K, Higashi M, et al. Clinical features of selenium deficiency in infants receiving long-term nutritional support. Nutrition 2007; 23: 782-787.

39. Fairweather-Tait SJ, Bao Y, Broadley MR, et al. Selenium in human health and disease. Antioxid Redox Signal 2011; 14: 1337-1383.

40. Wickett RR, Kossmann E, Barel A, et al. Effect of oral intake of cholinestabilized orthosilicic acid on hair tensile strength and morphology in women with fine hair. Arch Dermatol Res 2007; 299: 499-505.

41. Godfrey S, Staite W, Bowtell P, Marsh J. Metals in female scalp hair globally and its impact on perceived hair health. Int J Cosmet Sci 2013; 35: 264-271.

42. Wlaźlak E, Surkont G, Dunicz-Sokołowska A, et al. Analysis of calcium concentration in perimenopausal women hair. Prz Menopauzalny 2007; 1: $51-54$.

43. WHO/FAO. Vitamin and mineral requirements in human nutrition. Joint FAO/WHO Expert Consultation on Human Vitamin and Mineral Requirements. 2005.

44. Lima GP, Vianello F, Corrêa CR, et al. Polyphenols in fruits and vegetables and its effect on human health. Food and Nutr Sci 2014; 5: 1065-1082.

45. Zink A, Traidl-Hoffmann C. Green tea in dermatology - myths and facts. J Dtsch Dermatol Ges 2015; 13: 768-775.

46. Kumar N, Rungseevijitprapa W, Narkkhong NA. $5 \alpha$-reductase inhibition and hair growth promotion of some Thai plants traditionally used for hair treatment. J Ethnopharmacol 2012; 139: 765-771.

47. Pérez-Jiménez J, Neveu V, Vos F, Scalbert A. Identification of the 100 richest dietary sources of polyphenols: an application of the PhenolExplorer database. Eur J Clin Nutr 2010; 64: 112-120.

48. Peeters PH, Slimani N, van der Schouw YT, et al. Variations in plasma phytoestrogen concentrations in European adults. J Nutr 2007; 137: 1294-1300.

49. Kacalak-Rzepka A, Bielecka-Grzela S, Klimowicz A, et al. Dry skin as a dermatological and cosmetic problem. Ann Acad Med Stetin 2008; 54: 54-57.

50. Lengg N, Heidecker B, Seifert B, et al. Dietary supplement increases anagen rate in women with telogen effluvium: results of a randomized, placebocontrolled study. Therapy 2007; 4: 59-65.

51. Green AL, Weaver DF. Potential proconvulsant effects of oral zinc supplementation: a case report. Neurotoxicology 2008; 29: 476-477. 